\title{
An Overview of Hepatic Echinococcosis and the Characteristic CT and MRI Imaging Manifestations
}

\author{
Xiao-Kun Yu' \\ Le Zhang' \\ Wen-Jun $\mathrm{Ma}^{2}$ \\ Wen-Zhong $\mathrm{Bi}^{3}$ \\ Sheng-Gang Ju ${ }^{4}$ \\ 'Department of Radiology, The Fifth \\ Centre Hospital of Tianjin, Tianjin, \\ 300450, People's Republic of China; \\ ${ }^{2}$ Department of Radiology, Huangnan \\ Tibetan Autonomous Prefecture People's \\ Hospital, Huangnan, Qinghai Province, \\ 8I I300, People's Republic of China; \\ ${ }^{3}$ Department of Radiology, Qinghai \\ Tibetan Hospital, Xining, Qinghai \\ Province, 810000, People's Republic of \\ China; ${ }^{4}$ Department of Radiology, Henan \\ Mongol Autonomous County People's \\ Hospital, Xining, Qinghai Province, \\ 8II500, People's Republic of China
}

Correspondence: Le Zhang Department of Radiology, The Fifth Centre Hospital of Tianjin, No. 4I of Zhejiang Road, Binhai New District, Tianjin, 300450, People's Republic of China

Tel +86 $1065665539 ;+8613821510635$

Email zhangle824@outlook.com

\begin{abstract}
Hepatic echinococcosis is a parasitic, infectious disease with a high incidence in pastoral areas. It is highly infectious with a poor prognosis in some cases, which seriously affects the quality of life for people living in pastoral areas. This study aims to discuss the radiological characteristics, including computed tomography (CT) and magnetic resonance imaging (MRI), of hepatic echinococcosis from its definition, transmission, and pathological physiology. The characteristics of CT and MRI manifestations of cystic echinococcosis and alveolar echinococcosis are summarized in this study. It might help doctors to investigate this disease further and accurately make a diagnosis.
\end{abstract}

Keywords: hepatic echinococcosis, cystic echinococcosis, alveolar echinococcosis, brood capsule, daughter cyst, map sign, peninsula sign

\section{Introduction}

Echinococcosis is a severe zoonotic parasitic disease caused by larval forms of Echinococcus in animals and humans. ${ }^{1-3}$ The most common species encountered in humans are the Echinococcus granulosus, which causes cystic echinococcosis (CE), and the Echinococcus multilocularis, which causes alveolar echinococcosis (AE). ${ }^{2}$ Echinococcus granulosus is responsible for $95 \%$ of the human hydatid cases reported. $^{2}$ Although echinococcosis may involve any tissues or organs in the human body, the liver (50-77\%), lungs (15-47\%), spleen (0.5-8\%), and kidneys $(2-4 \%)$ are the most commonly involved. ${ }^{1-3}$

The disease continues to be a major public health issue in many regions of the world where agriculture and stockbreeding are primary sources of income. ${ }^{2}$ With increased globalization and human immigration, this regional disease may be widespread in certain places. In China, the parasitic disease survey suggests that patients with echinococcosis have been found in 23 provinces and autonomous regions, indicating that the distribution of echinococcosis is no longer limited to these northwestern pastoral areas (see Figure 1).

The prevalent population has a history of contact with nomadic infected areas, and the eggs excreted in the feces of the definitive host after being infected by the genus Echinococcus can be spread via animal activities, dust, wind, water, and other media. The eggs of Echinococcus are extremely resistant to low temperatures, dehydration, and chemicals, which is why echinococcosis is difficult to treat. ${ }^{4}$

This study aims to discuss the radiological characteristics, including computer tomography (CT) and magnetic resonance imaging (MRI), of hepatic echinococcosis from its definition, transmission, and pathological physiology. The 

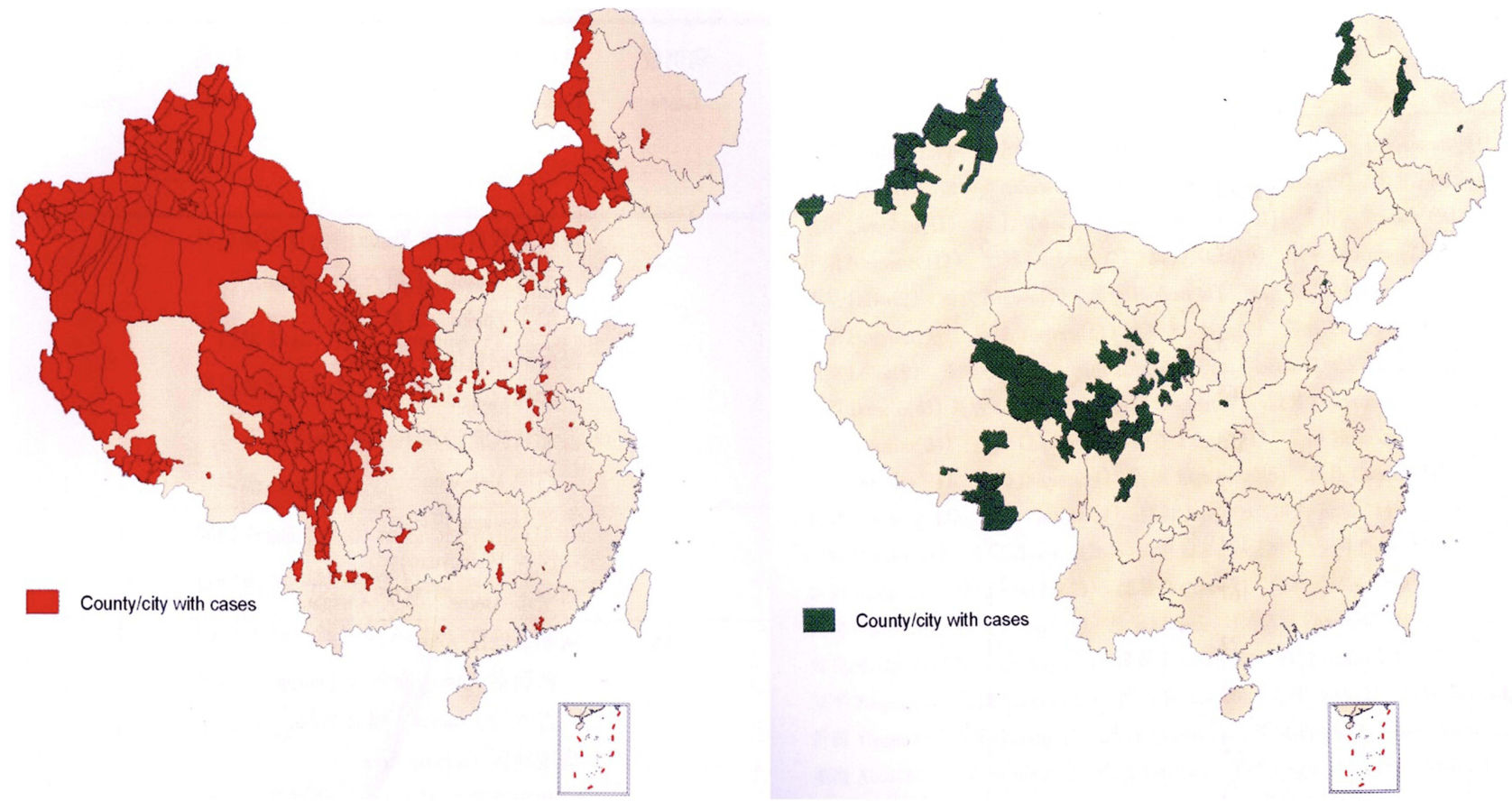

Figure I The epidemiological distribution of cystic and vesicular cysticercosis in China.

characteristics of CT and MRI manifestations of cystic echinococcosis and alveolar echinococcosis are summarized in this study. It might help doctors to investigate this disease further and accurately make a diagnosis.

\section{The Pathogens of Echinococcosis}

$\mathrm{CE}$ is the most common form and is caused by Echinococcus granulosus.

$\mathrm{AE}$ is considered the most dangerous parasitic disease and is caused by Echinococcus multilocularis (larvae). It is also known as the "worm cancer."

\section{The Source of Infection and Route of Transmission for Echinococcosis}

In the biological life cycle of echinococcosis, carnivores are the definitive hosts while herbivores are the intermediary hosts. ${ }^{2}$ Echinococcosis can be transmitted by dogs, wolves, foxes, jackals, etc. However, it is rare for wild animals to be a source of infection, and dogs are the main source of infection. $^{5}$ In the endemic areas, sheep and cattle often have echinococcosis, and residents often feed their dogs with the offal of sheep and other livestock, allowing these dogs the opportunity to ingest Echinococcus. The number of intestinal parasites can be in the hundreds or thousands after infection. The gravid proglottids are mobile and can attach to the dog's fur, and in some cases, they may cause itching in the anus when excreted. When the dog licks and bites, the proglottids are crushed, and the eggs in the feces can then contaminate the fur of the whole body. In the case of close contact with these dogs, humans are easily infected. Transmission can be direct or indirect. Direct infection occurs through close contact with a dog when the eggs transfer from the dog's fur to the hand and subsequently the mouth of the human. Indirect infection is caused by the contamination of food or water with eggs from the dog's feces, especially when humans and animals drink from the same water source. In dry and windy areas, eggs in the soil can be carried by the wind, leading to the possibility of secondary infection via the respiratory tract. ${ }^{6}$

\section{Clinical Meanings for Echinococcosis}

Hepatic echinococcosis (HE) accounts for approximately $70-80 \%$ of the total incidence of human cysticercosis, with CE being the most common, accounting for approximately $97 \%$ of this total. AE is extremely harmful, with the 10 -year mortality rate as high as $95 \%$ after onset. ${ }^{7}$ The liver is the most suitable organ for Echinococcus, and more than $70 \%$ of $\mathrm{CE}$ occurs in the liver, but it can involve multiple organs simultaneously. In AE cases, $100 \%$ are first found in the liver, and distant metastases can occur at an early stage. ${ }^{1}$ 
The definitive diagnosis of echinococcosis is based on imaging and serology, with ultrasound, which is radiationfree and less expensive than other imaging technologies, being the easiest method. In many parts of the world, portable ultrasound instruments are adopted as the primary screening tool. CT and MRI are popular in various regions, including some remote county hospitals in Tibetan areas that are equipped with MRI instruments. Characteristic manifestations of HE exist on CT and MRI, and these are increasingly being used in screening. ${ }^{8}$

\section{CE (Echinococcus granulosus) Epidemiological Investigations}

1. The definitive hosts: carnivores, such as dogs, wolves, and jackals.

2. The intermediate hosts: even-toed ungulates, such as sheep, cattle, camels, pigs, and deer, and occasionally, horses, kangaroos, some rodents, primates, and humans.

3. It is primarily transmitted via the sheep-dog and yak-dog chain and is most commonly found on the plateau in the northwestern pastoral regions.

4. Human infection is mainly through the ingestion of water, vegetables, or other food contaminated with the eggs of Echinococcus.

\section{Pathological Features: Cyst}

The early stage: The cyst is divided into an inner and outer layer with a characteristic double-walled structure. The inner layer is the intact parasite capsule membrane (the inner capsule). The outer layer refers to the reactive layer of compressed host tissue (the external capsule).

The degenerative stage: The cyst ruptures and collapses, and the inner capsule separates from the external capsule, with characteristic ribbon sign changes.

The middle and late stage: The cystic fluid is absorbed, and caseous changes and calcification of the cystic wall or cystic contents may occur.

All Echinococcus cysts are at risk of rupture, which is classified as a complication of $\mathrm{CE}$ and depends on factors such as age, chemical reaction, body defense mechanisms, the size and number of Echinococcus cysts, and the site of occurrence. $^{9}$

\section{Image Typing}

1. The simple cystic type: The typical double-layered structure of the cyst wall is visible, suggesting that the infection is in a biologically active phase (the early stage) at this time.
2. The multi-daughter cystic type: Echinococcus exhibits a strong regenerative capacity and biological activity, with imaging characteristics of multiple cysts (including daughter and granddaughter cysts).

3. The inner capsule detached type: Echinococcus exhibits degeneration and reduced biological activity, with shrinkage of the inner capsule and partial or complete detachment from the external capsule.

4. The solid calcification type: Echinococcus exhibits a gradual loss of activity and stability, with absorption and disappearance of the cystic fluid and partial or complete calcification.

5. The mixed type: This type is most often visible in different stages of infection or postoperative recurrence, with rupture of the cyst wall, co-infection, or calcification.

\section{Characteristic Imaging Manifestations}

$\mathrm{CE}$ is surrounded by an intact parasitic cystic membrane (the inner capsule) and a reactive layer of compressed host tissue (the external capsule). The cyst membrane produces cystic fluid, which expands the lesion distinctively in the periphery, and the daughter cysts give the cysts a multilocular character. The increased density of cystic fluid and calcification of the cyst wall/contents will occur in cases of cystic degeneration. When the inner capsule is detached from the external capsule, various signs of capsule detachment can appear (the floating band and the water lily sign). When the cyst ruptures, the cystic fluid and contents will leak into the bile duct or peritoneal cavity, causing implantation or metastasis (see Figure 2).

\section{Imaging Diagnostic Criteria}

The basic sign: The cyst.

Characteristic manifestations: (1) the cystic wall and calcification, (2) the daughter cyst sign, and (3) the cystic membrane detachment sign.

These diagnostic criteria need to be combined with a contact history in the endemic area and serological examination of echinococcosis.

Case 1 (see Figure 3): CE of the simple cystic type: A 31-year-old Tibetan female with a history of pastoral living, epigastric fullness, poor appetite, and weight loss. A single giant echinococcosis cyst is seen in the liver.

Case 2 (see Figure 4): CE of the multi-daughter cystic type: A 27-year-old Tibetan female with a history of pastoral living, weight loss, and a full abdomen. Multidaughter cysts are visible inside the big cyst. 

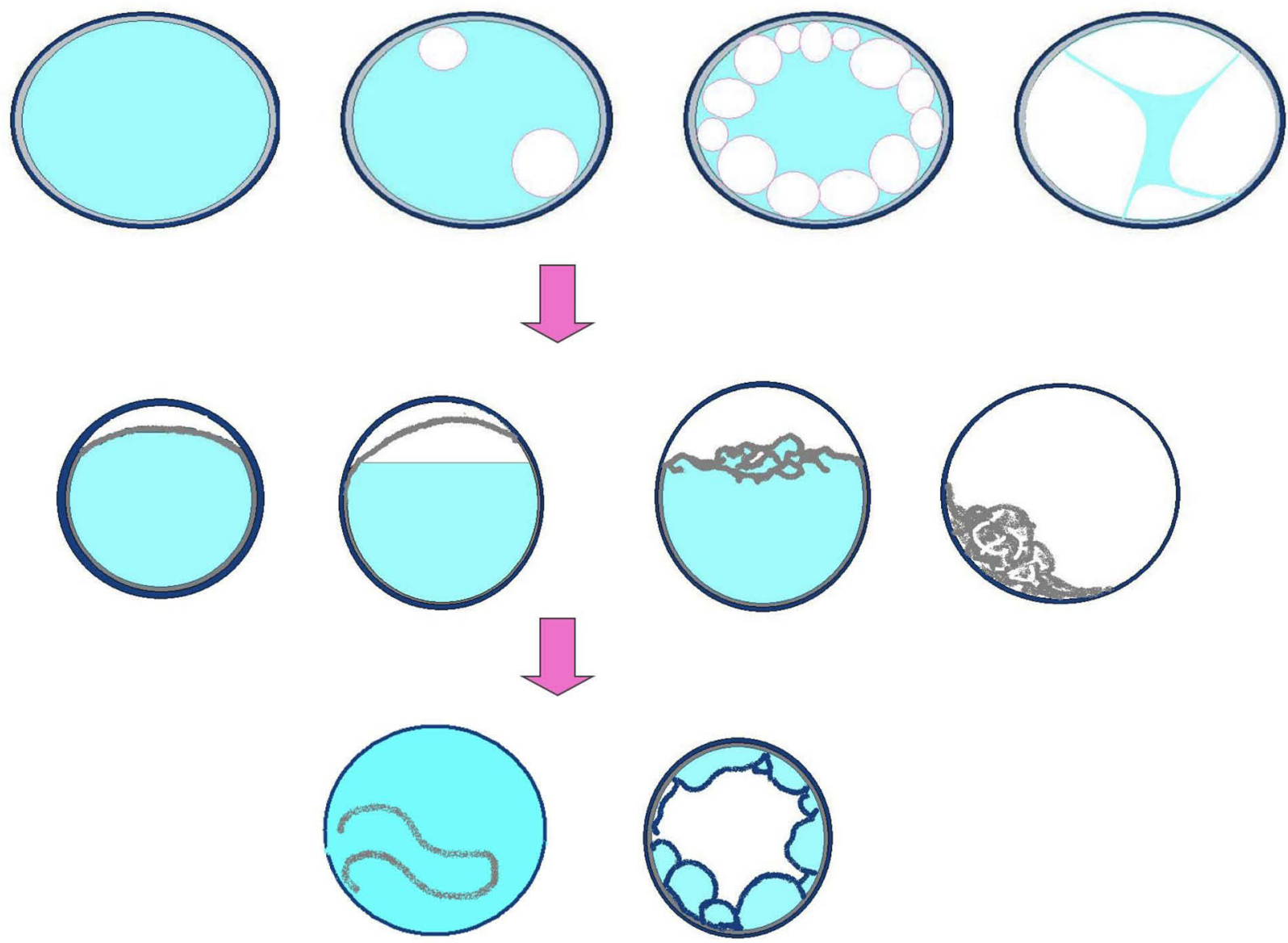

Figure 2 The pathological changes in cystic echinococcosis: When the course of echinococcosis was long, the external capsule of the Echinococcus often became thick and rough with calcium salt deposits or even completely calcified. The Echinococcus had mostly decayed and necrosed, with purulent cystic fluid. When the Echinococcus gradually degenerated with the absorption of the cystic fluid, the inner cyst wall folded and shrank, and the necrosis dissolved with caseous changes.

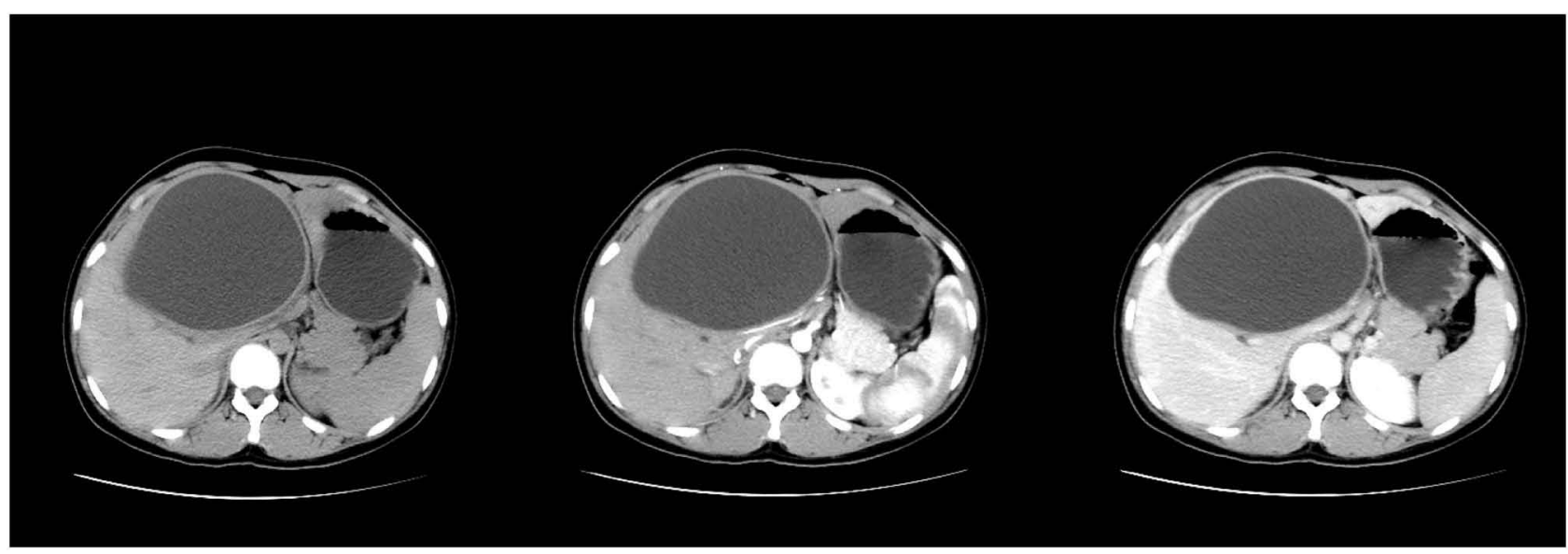

Figure 3 Cystic echinococcosis—-the simple cystic type. 


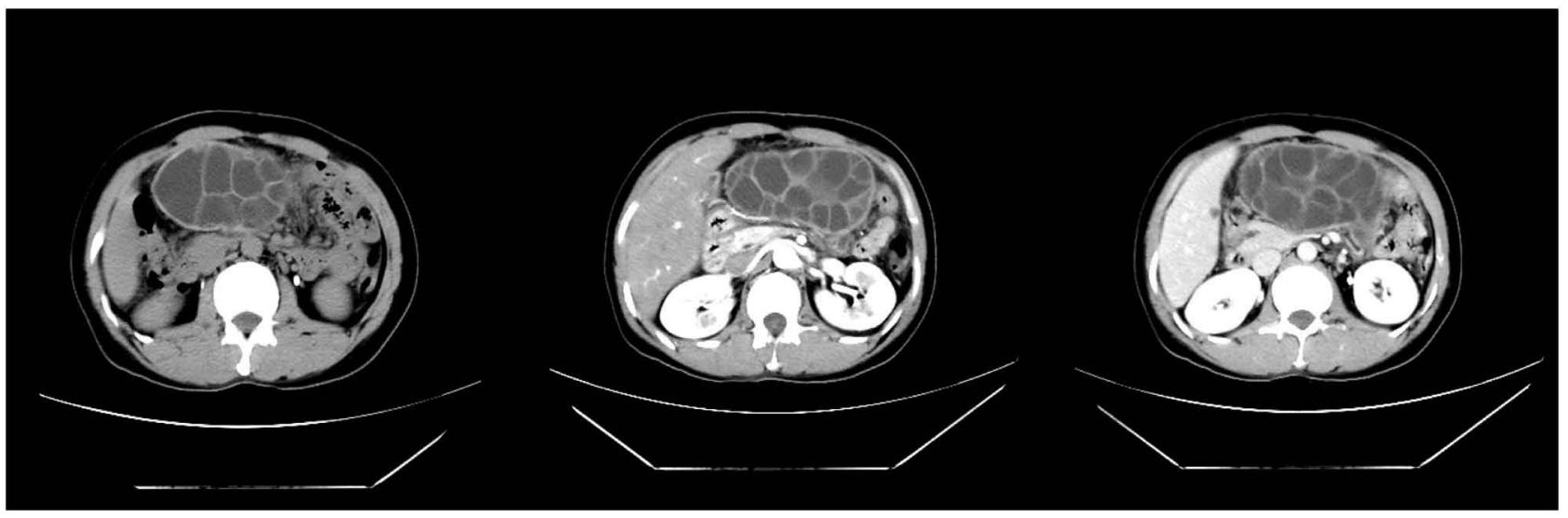

Figure 4 Cystic echinococcosis-the multi-daughter cystic type.

Case 3 (see Figure 5): CE of the detached inner capsule type: The inner capsule is collapsed and detached and is folded into a floating band sign.

Case 4 (see Figure 6): Postoperative recurrence of CE (rupture combined with infection): A 35-year-old Tibetan male with atrophy of the echinococcosis cyst, thickening of the cystic wall, and multiple gas shadows.

\section{AE (Echinococcus multilocularis) Epidemiological Investigations}

1. The definitive hosts: primarily foxes, but can also be dogs with complicated infections. ${ }^{5}$

2. The intermediate hosts: rodents or insectivores.

3. Since humans are not suitable intermediate hosts, when humans are infected, the capsules contain only jelly and no proto cercaria.

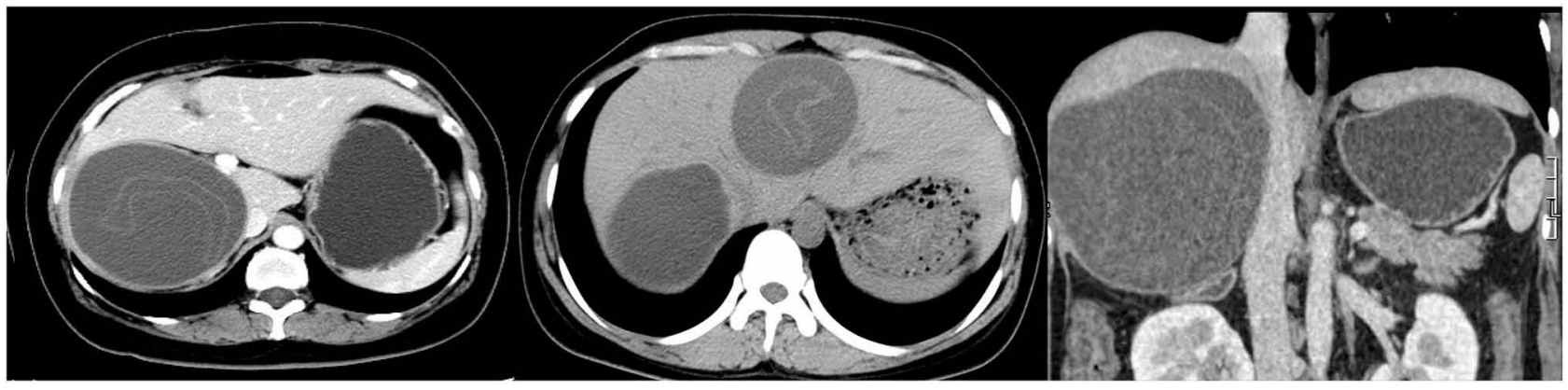

Figure 5 Cystic echinococcosis - the inner capsule detached type.

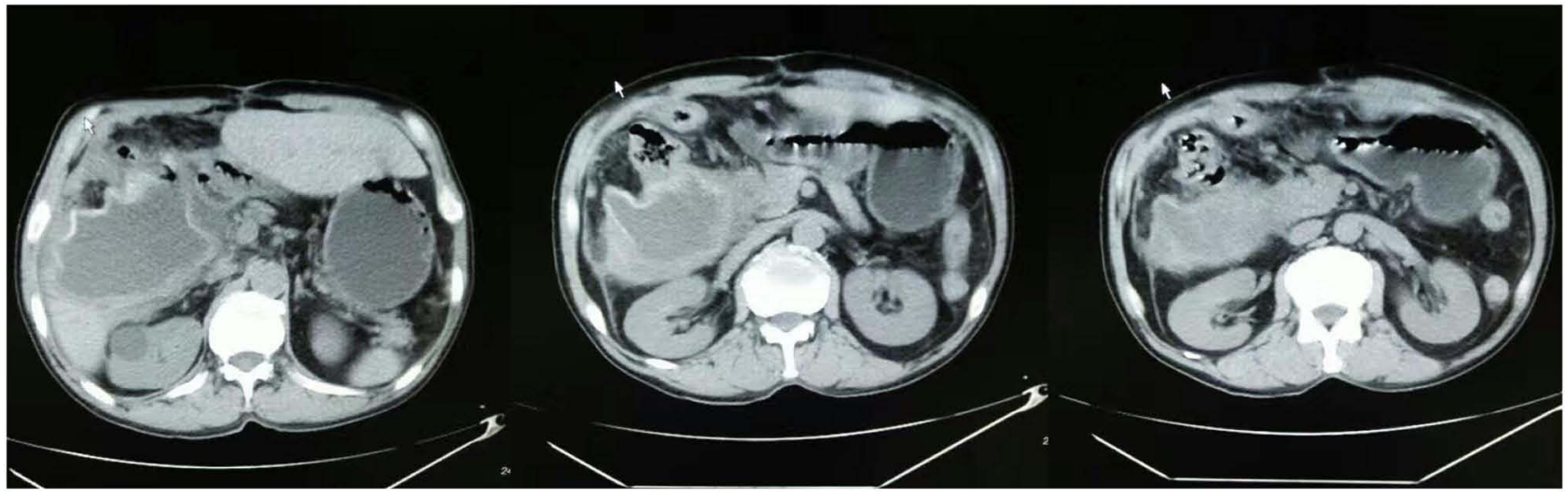

Figure 6 Cystic echinococcosis-postoperative recurrence, rupture, combined with infection. 
4. The endemic area is relatively small, mostly restricted to the areas where wild foxes and rodents are common.

5. It is very destructive to the human body, with $100 \%$ of cases affecting the liver and causing serious injury to hepatic functioning.

\section{Pathological Features: Solid Mass}

1. External bud proliferation of the larvae, with thousands of small alveolar larvae gathering into nodules, and infiltration of the surrounding tissues without restriction.

2. The enlargement of nodular lesions, fusing into irregular masses that are as hard as rubber and do not hemorrhage on cutting.

3. The internal necrosis and liquefaction of the lesion to form an effusion or cavity and a mass with multiple calcifications.

\section{Biological Characteristics}

The proliferation process of the larvae forms an infiltration zone, and three types of lesions, fibrosis, calcification, and liquefaction/cavitation, occur successively and coexist simultaneously as the lesion progresses.

\section{Imaging Diagnostic Criteria}

The basic sign: a heterogeneous parenchymal mass without enhancement.

Characteristic manifestations: small alveolar, calcification, and central liquefaction necrosis/cavity.

Important signs: The affected hepatic segment is reduced in size and contracted and depressed at the edges, while the normal hepatic segment compensates and is enlarged.

\section{Stages of $A E$}

The early stage: The lesions are limited to two hepatic segments.

The middle stage: The lesions exceed two hepatic segments.

The late stage: Complications of obstructive jaundice, portal hypertension, and multiple metastases occur.

\section{Characteristic Imaging Manifestations}

The calcification sign: Punctate calcification particles appear inside the lesion, with flocculate or irregularly large calcification foci.

The annual ring sign: The larvae proliferate on the periphery to form an infiltration zone, and calcium salt deposits appear in senescence and degeneration to form a layered calcification zone. Both the proliferative and degenerative pathological processes evolve and repeatedly expand, resulting in multilayered changes that resemble the appearance of an annual ring.

The internal trapping sign: As the length of time of infection increases, the larvae gradually decay, the necrotic area of the lesion becomes fibrotic, and the granular calcifications gradually fuse into large lamellar calcifications. Therefore, the exterior surface of the lesion appears contracted and depressed.

The cavity sign: The larvae proliferate into giant lesions with internal solidification, vascular occlusion, localized ischemic necrosis, and liquefaction into a jelly-like form with irregular location and morphology or with the appearance of crab-claw-like liquefied cavities.

The cave sign: On CT scanning, a low-density fluid cavity, which varies in size and shape and is surrounded by a calcified wall, appears within a high-density calcified lesion, forming a cave-like change.

The peninsula sign: In the case of a localized calcified mass in the cavity wall that protrudes into the cavity, a peninsula-like change is visible.

The vesicle sign: When the larvae persistently reproduce and infiltrate the surrounding hepatic tissues, small, honeycomb cyst-like structures, which represent strong biological activity, are visible.

The map sign: The cavity wall is bordered with hypertrophic fibrous tissue and is surrounded by bands of infiltration and calcification, resembling a map.

CT: A low-density infiltration zone is visible at the edge of the mass, coexisting with calcified deposits of irregular masses at the inner edge, and irregular cavities are mostly visible inside the mass.

MRI: The infiltration zone manifests as a low-signal irregular mass on T1- and T2-weighted images, and the inner edge of the fibrous zone manifests as a slightly hyperdense signal with localized invagination forming a peninsula sign and internal necrosis and liquefaction forming a fluid retention cavity.

The characteristic image is the infiltration zone around the mass, suggesting that the larvae are still living and proliferating, infiltrating the surrounding hepatic tissues, forming the halo sign, and spreading to the surrounding hepatic tissue in a crab-claw pattern. 


\section{Nine CT- or MRI-Specific Image Manifestations}

The halo, vesicle, calcification signs, the annual ring, internal trapping and cavity signs, the cave, peninsula, and map signs.

The diagnosis can be confirmed by any one of these signs, and the definitive diagnosis rate by imaging is $97 \%$ preoperatively. ${ }^{10}$

Case 5 (see Figure 7): AE with the annual ring sign.

Case 6 (see Figure 8): AE (CT imaging): A marginal, hypodense infiltration zone is observed, coexisting with both calcified deposits with irregular masses at the inner margin and a cavity (see Figure 8A). AE (MRI): The infiltration zone manifests as a hypodense irregular mass on T1- and T2-weighted images and the inner edge of the fibrous zone manifests as slightly hyperdense and locally invaginated to form a peninsula sign, with internal necrosis and liquefaction forming a fluid retention cavity (see Figures $8 \mathrm{~B}$ and $\mathrm{C}$ with enhancement).

Case 7 (see Figure 9): AE with the infiltration zone (vesicle sign) visible at the edge of the lesion, an irregular cavity inside the lesion, forming a map and a peninsula sign, and irregular calcifications.

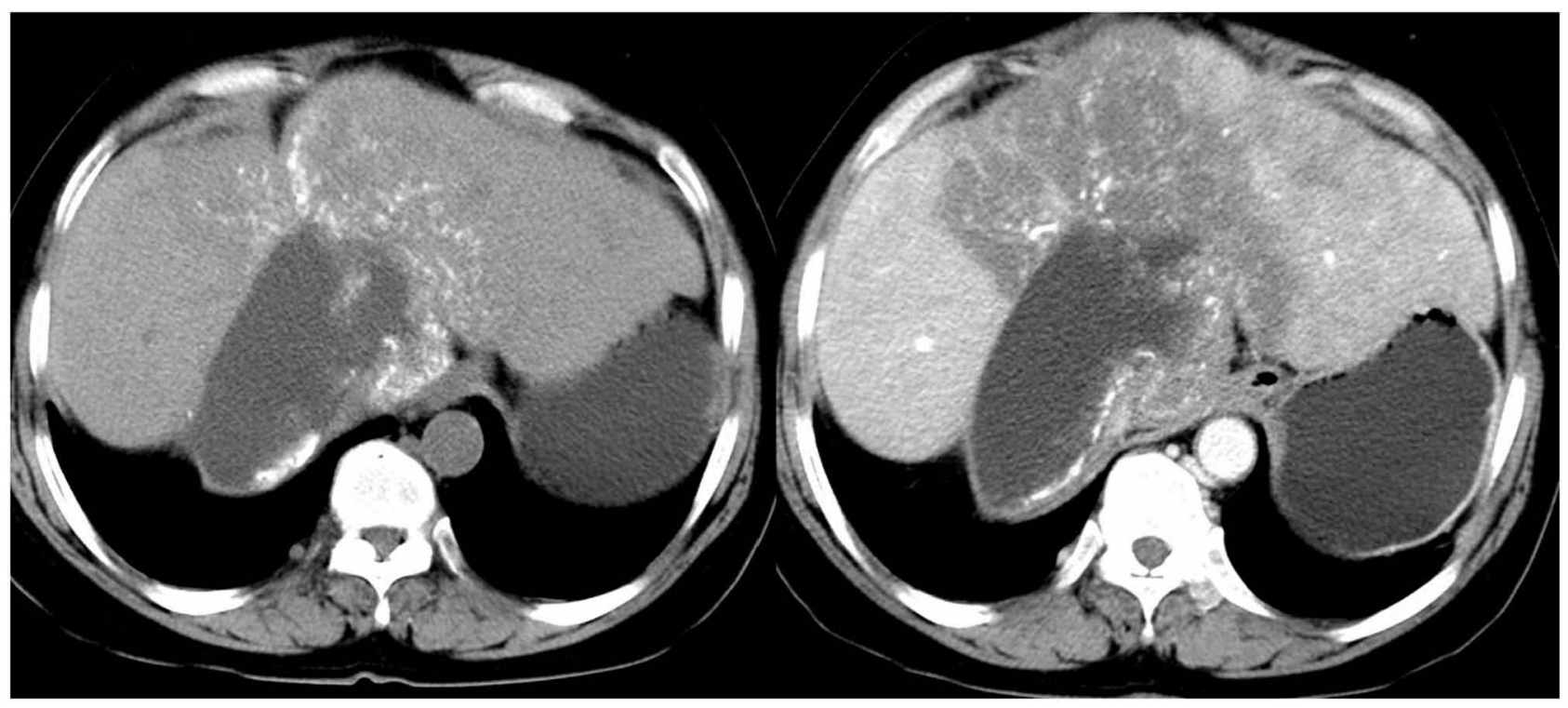

Figure 7 Alveolar echinococcosis-the annual ring sign.
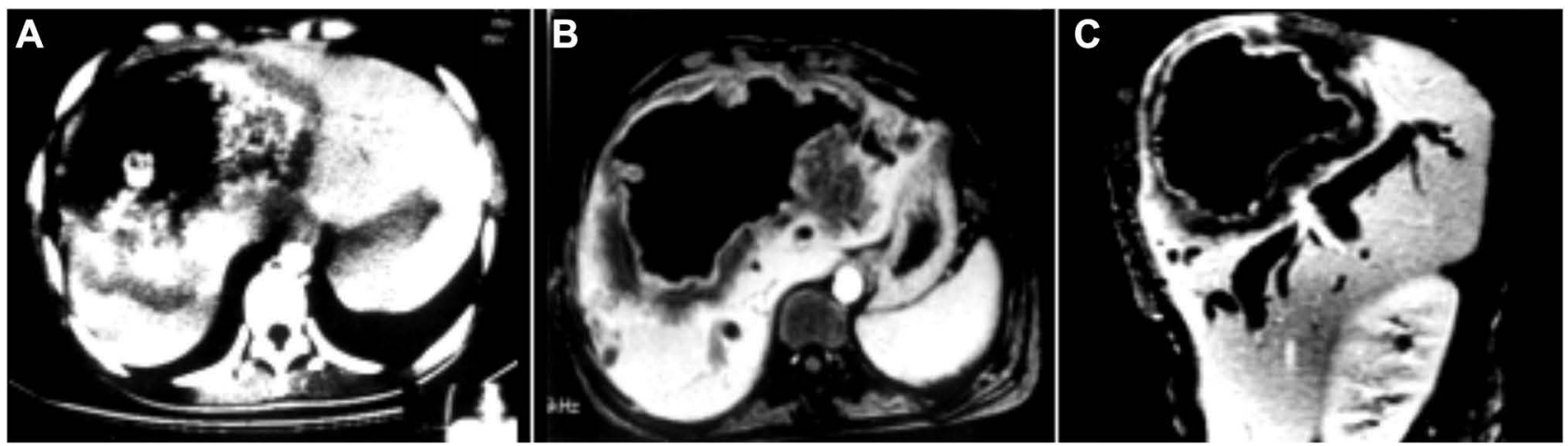

Figure 8 Alveolar echinococcosis - the CT and MRI manifestations. (A) CT imaging: A marginal, hypodense infiltration zone is observed, coexisting with both calcified deposits with irregular masses at the inner margin and a cavity. (B and C) MRI: The infiltration zone manifests as a hypodense irregular mass on TI- and T2-weighted images and the inner edge of the fibrous zone manifests as slightly hyperdense and locally invaginated to form a peninsula sign, with internal necrosis and liquefaction forming a fluid retention cavity. 

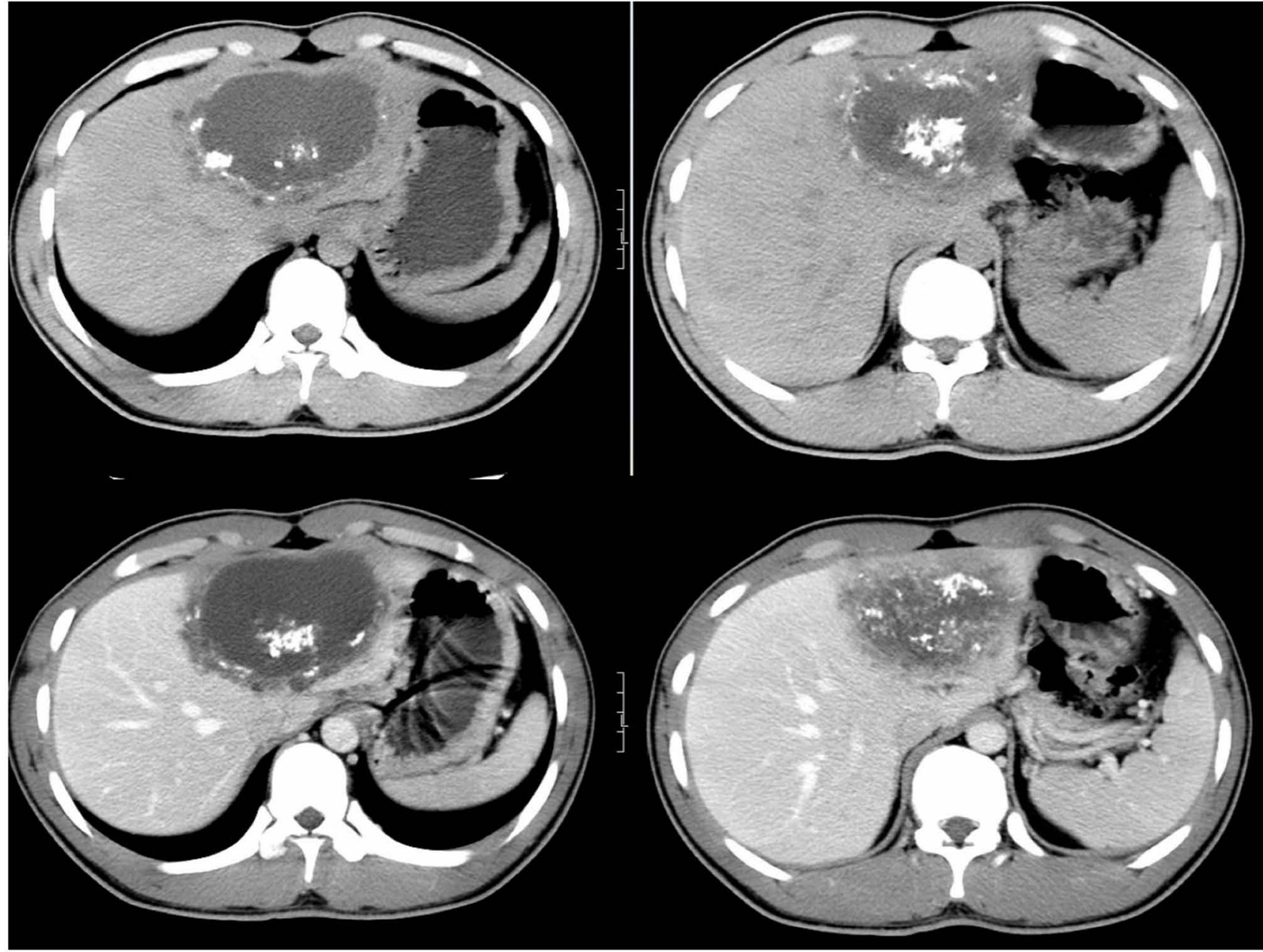

Figure 9 Alveolar echinococcosis—-the vesicle sign and peninsula sign.

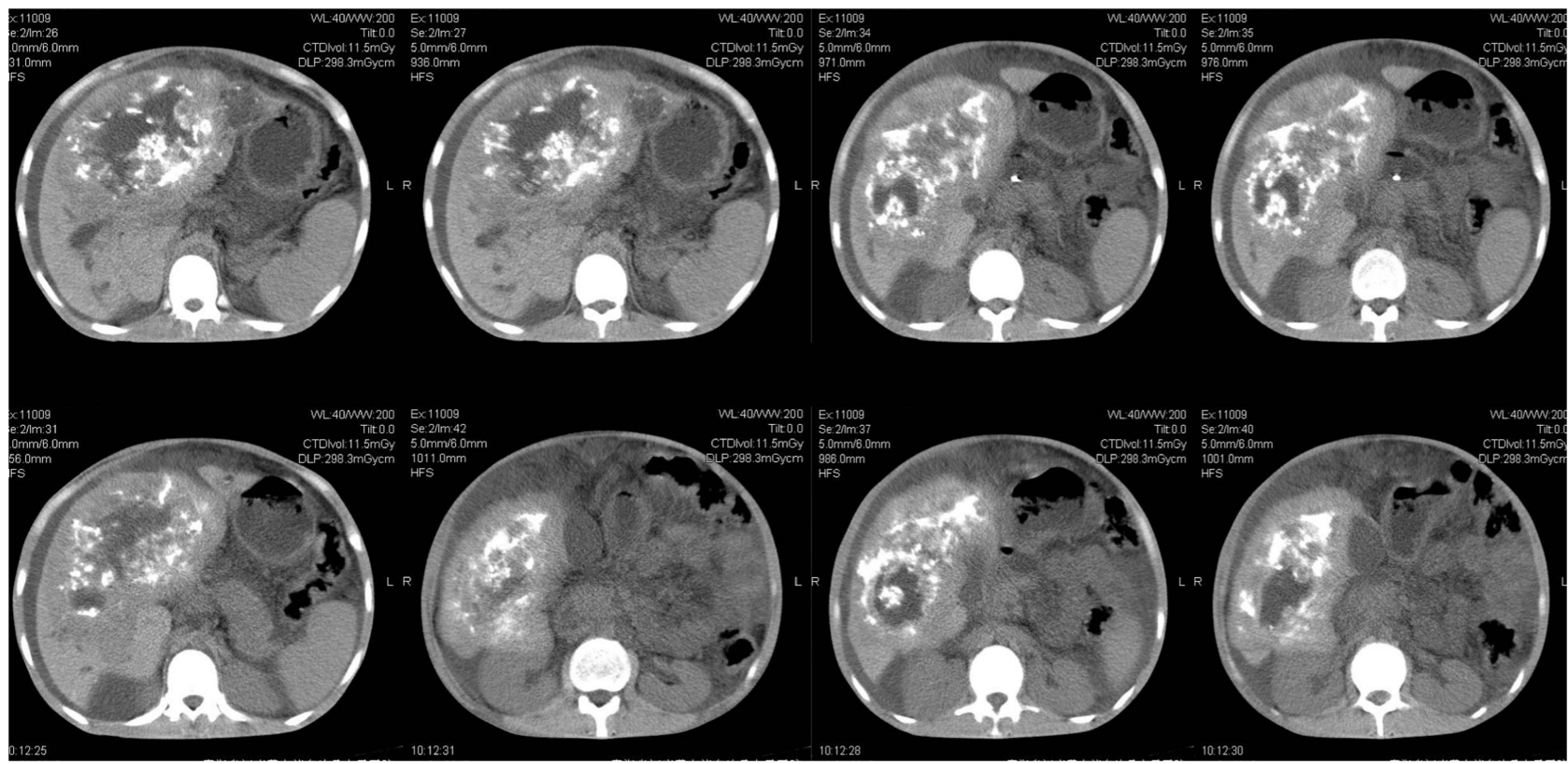

Figure 10 Alveolar echinococcosis—with multiple typical signs. 
Case 8 (see Figure 10): AE with multiple typical signs.

\section{Summary}

\section{The Images of CE and AE Have}

\section{Characteristic Lesions, and the Main} Points of Differentiation are as Follows

The characteristic signs of CE: smooth and neat edges and clear and sharp demarcation, similar to the appearance of an orange.

1. The cystic wall: thick wall, double-wall, and eggshell signs.

2. The daughter cysts: cyst-in-cyst grape, honeycomb, and wheel signs.

3. The rupture of the cyst: sky screen, folding, water snake, streamer, gyri, and consolidation signs.

The characteristic signs of AE: infiltrative growth with indistinct borders, resembling a cactus.

1. The infiltrative proliferation: vesicle, halo zone, and annual ring signs.

2. The fibrous calcification: consolidation, invagination, and granular/large calcification signs.

3. The liquefaction cavity: cave, peninsula, and map signs.

\section{Funding}

There is no funding to report.

\section{Disclosure}

The authors declare that they have no competing interests.

\section{References}

1. Ma L, Chen DC, Zou SY, Liu YY, Zhou LY, Xiu ZG. Epidemiological characteristics of hepatic echinococcosis, concurrent cerebral echinococcosis, and pulmonary echinococcosis in Ganzi County, Sichuan Province, China. Medicine. 2020;99:e19753. doi:10.1097/MD.0000000000019753

2. Akbulut S, Yavuz R, Sogutcu N, et al. Hydatid cyst of the pancreas: report of an undiagnosed case of pancreatic hydatid cyst and brief literature review. World J Gastrointest Surg. 2014;6:190-200. doi:10.4240/wjgs.v6.i10.190

3. Akbulut S. Parietal complication of the hydatid disease: comprehensive literature review. Medicine. 2018;97:e10671. doi:10.1097/ MD.0000000000010671

4. Cheng SL, Wang H, Ma X, et al. [An epidemiological survey on echinococcosis in yushu prefecture of Qinghai Province]. Zhongguo Ji Sheng Chong Xue Yu Ji Sheng Chong Bing Za Zhi. 2016;34:547-551. Chinese.

5. Weng $\mathrm{X}, \mathrm{Mu} \mathrm{Z}$, Wei $\mathrm{X}$, et al. The effects of dog management on Echinococcus spp. prevalence in villages on the eastern Tibetan Plateau, China. Parasit Vectors. 2020;13:207. doi:10.1186/s13071020-04082-6

6. Hui L, Ning X, Shi-Jie Y, Dong W, Jia P. [Epidemiological characteristics of canine Echinococcus infection in Qinghai-Tibet Plateau of China]. Zhongguo Xue Xi Chong Bing Fang Zhi Za Zhi. 2017;29:129-138. Chinese.

7. Craig PS, Giraudoux P, Wang ZH, Wang Q. Echinococcosis transmission on the Tibetan Plateau. Adv Parasitol. 2019;104:165-246.

8. Armoon A, Mehrian P, Soleimantabar H, Torabi S, Ghahderijani BH. Computer Tomography (CT) characteristics of pulmonary cystic echinococcosis. Med Arch. 2019;73:338-343. doi:10.5455/ medarh.2019.73.338-343

9. Garg MK, Sharma M, Gulati A, et al. Imaging in pulmonary hydatid cysts. World J Radiol. 2016;8:581-587. doi:10.4329/wjr. v8.i6.581

10. Jun-Hu B, Yong-Hai Z, Xiu-Min H, Yao-Dong L, Yong-Xing D. [Imaging analysis of 79 cases of hepatic echinococcosis]. Zhongguo Xue Xi Chong Bing Fang Zhi Za Zhi. 2019;30:674-677. Chinese.
Infection and Drug Resistance

\section{Publish your work in this journal}

Infection and Drug Resistance is an international, peer-reviewed openaccess journal that focuses on the optimal treatment of infection (bacterial, fungal and viral) and the development and institution of preventive strategies to minimize the development and spread of resistance. The journal is specifically concerned with the epidemiology of antibiotic resistance and the mechanisms of resistance development and diffusion in both hospitals and the community. The manuscript management system is completely online and includes a very quick and fair peerreview system, which is all easy to use. Visit http://www.dovepress.com/ testimonials.php to read real quotes from published authors. 